\title{
Response to single agent PD-1 inhibitor after progression on previous PD-1/PD-L1 inhibitors: a case series
}

\author{
Dylan J. Martini ', Aly-Khan A. Lalani', Dominick Bossé ${ }^{1}$, John A. Steinharter ${ }^{1}$, Lauren C. Harshman', \\ F. Stephen Hodi ${ }^{2,3}$, Patrick A. Ott ${ }^{2,3}$ and Toni K. Choueiri ${ }^{*}$
}

\begin{abstract}
Background: Monoclonal antibodies targeting the PD-1/PD-L1 axis have gained increasing attention across many solid tumors and hematologic malignancies due to their efficacy and favorable toxicity profile. With more than 1 agent now FDA-approved in a wide variety of tumor types, and with others in clinical trials, it is becoming more common that patients present to clinic for potential treatment with a second PD-1/PD-L1 inhibitor.

Case presentation: In this report, we present two patients with renal cell carcinoma and one with melanoma who received PD-1/PD-L1 inhibitors. Upon progression on their first-line PD-1/PD-L1 inhibitors, these patients received a different PD-1 inhibitor (nivolumab in all cases) and all had progressive disease as their best response to the subsequent PD-1 inhibitor. The reported clinical information focuses on the course of the disease and the responses to all treatment regimens.

Conclusions: Clinicians should refrain from using multiple PD-1/PD-L1 inhibitors sequentially outside of clinical trials until there is sufficient data to support this practice routinely. Prospective studies that allow prior treatment with PD-1/PD-L1 are needed to validate the efficacy and safety of these drugs in the second line or later setting. Furthermore, ongoing efforts that aim to identify mechanisms of resistance to immunotherapy will be informative and may ultimately assist physicians in select the optimal treatment following progression on PD-1/PD-L1 inhibitor.
\end{abstract}

Keywords: PD-1/PD-L1 inhibitor, Sequential treatment, Immune checkpoint blockade, Case reports

\section{Background}

The programmed cell death protein-1 (PD-1) and its ligands, PD-L1 and PD-L2, are part of a pathway that cancer cells utilize to evade immune surveillance [1]. Monoclonal antibodies targeting the PD-1/PD-L1 axis have demonstrated efficacy in numerous malignancies [2-16]. Five of these agents (atezolizumab, nivolumab, pembrolizumab, avelumab, and durvalumab) have gained United States Food and Drug Administration (FDA) approval for the treatment of non-small cell lung cancer (NSCLC), renal cell carcinoma (RCC), melanoma, urothelial carcinoma, head and neck squamous cell carcinoma (HNSCC), Hodgkin's Lymphoma, and Merkel

\footnotetext{
* Correspondence: Toni_Choueiri@dfci.harvard.edu

${ }^{1}$ Lank Center for Genitourinary Oncology, Dana-Farber Cancer Institute,

Harvard Medical School, 450 Brookline Ave D1230, Boston, MA 02215, USA

Full list of author information is available at the end of the article
}

cell carcinoma [17]. With these agents and others in development, physicians are more commonly faced with the question of whether to treat patients with sequential PD-1 blockade. While there is a general acceptance that these drugs are "similar", there are some subtle differences among them and many clinicians wonder if a subsequent different PD-1 inhibitor can of be of any help to patients with few therapeutic options after progression. The outcomes of these patients in this expanding clinical setting are largely unknown and have not been assessed in clinical trials. In this report, we present the cases of three patients (two with metastatic RCC and one with melanoma) who initially responded to PD-1/PD-L1 blockade before progressing and later immediately progressed upon re-treatment with a different PD-1 inhibitor. 


\section{Case presentations}

\section{Case presentation 1}

A 54-year-old man underwent a radical nephrectomy which revealed an $11.5 \mathrm{~cm}$ pT2bN0M0 clear cell renal cell carcinoma (ccRCC) on pathologic review. Four years later, he was diagnosed with metastatic disease to the lungs and hilar lymph nodes and began treatment with an anti-PD-L1-based combination. The patient had a best response of stable disease with tumor shrinkage and remained on treatment for 15 months. He discontinued therapy for progressive disease to the sacrum and the cerebellum and subsequently underwent stereotactic radiosurgery to the brain. Approximately 7 weeks after the last dose of the anti-PD-L1-based combination, he initiated treatment with $3 \mathrm{mg} / \mathrm{kg}$ nivolumab monotherapy every 2 weeks. After the patient received 4 doses, imaging showed progressive disease in the lung and hilar lymph node after 7 weeks.

\section{Case presentation 2}

A 67-year-old male was diagnosed with pT2aN0M1 ccRCC with multiple subcentimeter metastases to the lungs. The patient initially underwent metastatectomy to remove a $0.6 \mathrm{~cm}$ tumor in the left upper lobe, but he experienced progression 1 year later. An anti-PD-L1-based combination was started and he had stable disease as best response with tumor shrinkage and remained on treatment for 8 months until he discontinued therapy for new liver metastases. He then progressed after 2 cycles of axitinib. The patient received 8 doses of $3 \mathrm{mg} / \mathrm{kg}$ nivolumab monotherapy every 2 weeks, 6 months after the last dose of the anti-PD-L1based combination. He experienced disease progression in the lung, lymph nodes, and liver after 4 months.

\section{Case presentation 3}

A 78-year-old gentleman was diagnosed with stage IVM1c BRAF ${ }^{\mathrm{V} 600}$ mutant cutaneous melanoma with metastases to the kidney, adrenal, and lymph node. The patient began treatment with a vemurafenib, a BRAF inhibitor, but discontinued after two months for progressive disease. He then progressed through treatment with cytotoxic chemotherapy, ipilimumab, and a combination of anti-BRAF/ MEK (dabrafenib plus trametinib) combination therapy. He later received therapy with anti-PD-1 pembrolizumab for 5 months before being discontinued for treatmentrelated insulin-dependent diabetes mellitus, chronic pruritus, and joint pain. The patient had a complete response on treatment. Approximately 20 months after the patient's last dose of pembrolizumab, he progressed and initiated nivolumab every 2 weeks for 3 doses. Although the patient continued on insulin for treatment of diabetes mellitus throughout his time on nivolumab, neither the pruritus nor the joint pain recurred after treatment with nivolumab. Treatment was discontinued after 7 weeks due to disease progression in the bilateral adrenal lesions, lymph nodes, and a new lesion in the liver.

\section{Discussion}

Given the approval of different PD-1 and PD-L1 inhibitors in several solid tumor and hematological malignancies as of May 2017, it is anticipated that clinical decisions facing patients and physicians as described above will become more common. For example, there are already two PD-1 inhibitors, pembrolizumab and nivolumab, approved for NSCLC and melanoma in both the first and second-line setting. The shifting treatment paradigms in NSCLC and other diseases such as melanoma and RCC are complicated by the paucity of data for patients who fail PD-1/ PD-L1 inhibitors. This is partly because randomized trials in which these agents were investigated in the second-line setting or beyond, did not allow inclusion of patients who had prior treatment with a PD-1 or PD-L1 inhibitor $[3-10,12,13,15,18]$. Currently, there are numerous ongoing and pending trials in RCC, melanoma, and NSCLC that allow prior treatment with a PD-1/PDL1 inhibitor (Table 1). As many of these agents target the same PD-1/PD-L1 axis, development of resistance to one agent may plausibly lead to class resistance and, therefore, the likelihood of response to a second monotherapy agent given from the same class could be low.

When successful, PD-1 blockade establishes a favorable equilibrium between the immune response to, and immune evasion by, cancer cells [19]. In some situations, patients who achieve an objective response on treatment eventually experience disease progression. This acquired resistance to immunotherapy, also known as secondary tumor immune escape, has many plausible immunological explanations such as decreased intratumoral T-cell infiltration, suboptimal T-cell activation, upregulation of additional immune-checkpoints, or loss of tumor immunogenicity $[20,21]$. A recent genomic analysis comparing pretreatment and progression biopsy samples from melanoma patients who initially experienced an objective response on anti-PD-1 therapy showed that resistance was associated with: diminished antigenpresentation due to a defective beta-2-microglubulin (B2M) protein or impaired interferon-receptor signaling due to truncated Janus Kinase 1 (JAK1) or Janus Kinase 2 (JAK2) proteins [22]. Another study comparing differentially expressed genes between responders and non-responders to immunotherapy in melanoma showed that non-responders express a transcriptional signature exhibiting simultaneous upregulation of genes involved in the regulation of angiogenesis, cell adhesion, mesenchymal transition, extracellular matrix (ECM remodeling), and wound-healing [23]. 
Table 1 Select ongoing and pending clinical trials that allow prior treatment with a PD-1 or PD-L1 inhibitor

\begin{tabular}{|c|c|c|c|c|}
\hline Clinicaltrials.gov identifier & Experimental agents & Regimen targets & Phase & Tumor type \\
\hline NCT02899078 & Nivolumab + Ibrutinib & PD-1 + BTK & $1 \mathrm{~b} / 2$ & RCC \\
\hline NCT02923531 & Nivolumab + X4P-001 & PD-1 + CXCR4 & $1 b / 2 a$ & $\mathrm{RCC}$ \\
\hline NCT02963610* & Pembrolizumab + Lenalidomide & PD-1 + immunomodulatory & $1 / 2$ & NSCLC \\
\hline NCT03083808 & Pembrolizumab + Docetaxel/Pemetrexed/Gemcitabine & PD-1 + Chemotherapy & 2 & NSCLC \\
\hline NCT03041181 & Nivolumab + Docetaxel & PD-1 + Chemotherapy & 2 & NSCLC \\
\hline NCT02437136 & Pembrolizumab + Entinostat & PD-1 + HDAC & $1 \mathrm{~b} / 2$ & NSCLC and melanoma \\
\hline NCT02959437 & Pembrolizumab + Azacitidine + Epacadostat & PD-1 + Chemotherapy + IDO-1 & $1 / 2$ & NSCLC ${ }^{* *}$ \\
\hline NCT03084640 & Pembrolizumab + CMP-001 & PD-1 + TLR9 & $1 \mathrm{~b} / 2$ & melanoma \\
\hline NCT03014648 & Atezolizumab & PD-L1 & 2 & $\mathrm{NSCLC}$ \\
\hline
\end{tabular}

PD-1 programmed cell death protein 1, BTK: Bruton's tyrosine kinase, RCC: renal cell carcinoma, CXCR4: C-X-C chemokine receptor type 4, NSCLC: non-small cell lung cancer, HDAC: histone deacetylases, IDO-1: Indoleamine 2,3-Dioxygenase 1, TLR9: Toll-like receptor 9, PD-L1 programmed death-ligand 1

*Prior treatment with anti-PD-1/PD-L1 is only allowed for the Phase 2 portion of this trial (which is only for NSCLC patients)

${ }^{* *}$ Only the NSCLC cohort allows prior treatment with PD-1/PD-L1

Please note that this table was constructed using the following search terms on clinicaltrials.gov on 5/12/2017: "Nivolumab AND previously treated",

"Pembrolizumab AND previously treated", and "Atezolizumab AND previously treated". After generating a list of trials, the eligibility criteria for each trial was

manually screened for inclusion in this table

Although many patients experience resistance after responding to PD-1 or PD-L1 inhibitors, there may be situations where re-treatment has plausible rationale. Re-treatment with PD-1 blockade may be especially effective in patients who discontinue the first PD-1 or PD-L1 inhibitor for reasons other than disease progression. In fact, successful re-introduction of anti-PD-1 therapy after delayed tumor recurrence has been reported in one patient with melanoma [24]. Data have shown that even similar PD-1 agents, such as pembrolizumab and nivolumab, target different epitopes of PD-1 [25]. It is also reasonable to treat a patient with a PD-1 inhibitor after a PD-L1 inhibitor, or vice versa, given that the PD-1 pathway has 3 inhibitory interactions (PD1:PD-L1, PD-1:PD-L2, and PD-L1:B7.1) and each of these drugs only inhibit two of these [20]. A melanoma mouse model has shown that there is a synergistic effect of combining PD-1 and PD-L1 inhibition [26]. There is also an ongoing clinical trial testing the combination of PD-1 and PD-L1 inhibition [NCT02118337].

There are numerous opportunities to address mechanisms of resistance to immunotherapy other than retreatment with PD-1/PD-L1 monotherapy. For example, anti-Vascular Endothelial Growth Factor (VEGF) therapy has been shown to increase T-cell infiltration [27] and preclinical data suggests that antibodies blocking T-cell co-stimulatory proteins [28-30] or low-dose Interleukin-2 (IL-2) [31] may augment the immune response to anti-PD-1/PD-L1. Currently, clinical trials are ongoing which investigate Lymphocyte-activation gene 3 (LAG-3) and T-cell immunoglobulin and mucin-domain containing-3 (TIM-3) blockade either as monotherapy or in combination with an anti-PD-1 agent [NCT0 1968109, NCT02676869, NCT02817633, NCT02936102, NCT02966548, NCT02460224, NCT03005782]. There is also an early clinical trial investigating the synergy of IL2 and PD-1 inhibition in metastatic RCC [NCT02989714]. Additionally, stereotactic radiation therapy has been shown to increase the repertoire of antigen-specific T-cells and antitumor antibodies, which may increase the likelihood of the "abscopal effect" [32]. This suggests a possible synergy between radiotherapy and immunotherapy, which is being investigated in several ongoing clinical trials [NCT02821182, NCT0 2608385, NCT03050554, NCT02407171, NCT02843165, NCT02837263].

However, it is unlikely possible to provide clinical trials for all potential immunotherapy sequences. Therefore, databases that captures real-life clinical data on the safety and efficacy of anti-PD-1/PD-L1 therapy after treatment with another PD-1/PD-L1 inhibitor may potentially help to better characterize the outcomes of these patients in an unselected setting. For example, there is a United States-based registry for patients treated with high-dose IL-2, Proleukin ${ }^{\bullet}$ Observational Registry to Evaluate the Treatment Patterns and Clinical Response in Malignancy (PROCLAIM), which adds realworld experience for patients treated with IL-2 [33]. Similar registries for anti-PD-1/PD-L1 would also be beneficial in the current immunotherapy era.

\section{Conclusions}

Currently, there is insufficient data to support treatment with a different PD-1/PD-L1 inhibitor after progression on PD-1 pathway blockade, and such practice should be discouraged. Ideally, prospective studies that investigate the efficacy and safety of a PD-1/PD-L1 inhibitor in the second-line setting or later, which allows prior treatment with one of these agents, are needed to validate the use of sequential PD-1 blockade. Combined strategy such as dual 
immune agents or combination of PD-1/PD-L1 nonimmune therapy appears to be more supported by preclinical data and such trials represent a better opportunity to improve outcomes. Furthermore, ongoing efforts that aim to identify mechanisms of resistance to immunotherapy will be informative and may ultimately assist physicians in selecting a rational approach to the initial, and sequential, treatment options for these patients.

\section{Abbreviations}

B2M: Beta-2-microglubulin protein; ccRCC: Clear cell renal cell carcinoma; ECM: Extracellular matrix; FDA: United States Food and Drug Administration; HNSCC: Head and neck squamous cell carcinoma; IL-2: Interleukin-2; LAG-3: Lymphocyte-activation gene 3; NSCLC: Non-small cell lung cancer; PD-1: Programmed cell death protein-1; PD-L1: Programmed death ligand-1; PD-L2: Programmed death ligand-2; PROCLAIM: Proleukin ${ }^{\circledR}$ Observational Registry to Evaluate the Treatment Patterns and Clinical Response in Malignancy; RCC: Renal cell carcinoma; TIM-3: T-cell immunoglobulin and mucin-domain containing-3; VEGF: Vascular endothelial growth factor

\section{Acknowledgements}

Not applicable.

\section{Funding}

This research was supported in part by the Dana-Farber/Harvard Cancer Center Kidney SPORE, and the Trust Family, Michael Brigham, and Loker Pinard Funds for Kidney Cancer Research at Dana-Farber Cancer Institute for Toni K. Choueiri.

\section{Availability of data and materials}

Not applicable.

\section{Authors' contributions}

DJM was involved in the identification and selection of patient cases and drafted the manuscript. AAL and DB were involved in the drafting and editing of the manuscript. JAS, LCH, FSH reviewed and edited the manuscript. PAO and TKC were involved in the identification, selection, and management of patient cases and reviewed and edited the manuscript. All authors read and approved the final manuscript.

\section{Ethics approval and consent to participate}

Not applicable.

\section{Consent for publication}

Written informed consent was obtained from the patients for publication of their individual details. The consent forms are held by the authors and are available for review by the Editor-In-Chief.

\section{Competing interests}

Lauren C. Harshman is on the advisory board of Genentech, Pfizer, Dendreon, NCCN, Medivation, Kew and has received research funding/ support from Bayer, Medivation/Astellas, Pfizer, Dendreon, Sotio, Genentech, Merck, BMS, Jannsen. F. Stephen Hodi has a consulting or advisory role with Amgen, Genentech/Roche, Merck Sharp \& Dohme, and Novartis. He has received research funding from Bristol-Myers Squibb (Inst), Genentech/Roche (Inst), Merck Sharp \& Dohme (Inst), and Novartis (Inst). He has a patent pending as per institutional policy and patent pending royalties received on MICA related disorders application to institution per institutional IP policy. He has received travel accommodations and expenses from Bristol-Myers Squibb and Novartis. He has a relationship with Bristol-Myers Squibb and Genentech/Roche. Patrick A. Ott has a consulting or advisory role with Alexion Pharmaceuticals, Amgen, Bristol-Myers Squibb, Celldex, CytomX Therapeutics, Genentech and Neon Therapeutics. He has received research funding from ARMO BioSciences (Inst), AstraZeneca/Medlmmune (Inst), Bristol-Myers Squibb (Inst), Celldex (Inst), Merck (Inst). Toni K. Choueiri has received institutional research funding from Pfizer, Exelixis, BMS, Novartis, and has an advisory role at Pfizer, Novartis, Genentech, Merck, BMS and Bayer. The remaining authors have no disclosures.

\section{Publisher's Note}

Springer Nature remains neutral with regard to jurisdictional claims in published maps and institutional affiliations.

\section{Author details}

${ }^{1}$ Lank Center for Genitourinary Oncology, Dana-Farber Cancer Institute, Harvard Medical School, 450 Brookline Ave D1230, Boston, MA 02215, USA. ${ }^{2}$ Melanoma Center, Dana-Farber Cancer Institute, Harvard Medical School, Boston, MA, USA. ${ }^{3}$ Center for Immuno-Oncology, Dana-Farber Cancer Institute, Harvard Medical School, 450 Brookline Ave, Boston, MA 02215, USA.

Received: 1 June 2017 Accepted: 1 August 2017

Published online: 15 August 2017

\section{References}

1. He J, et al. Development of PD-1/PD-L1 pathway in tumor immune microenvironment and treatment for non-small cell lung cancer. Sci Rep. 2015;5:13110

2. Balar AV, et al. Atezolizumab as first-line treatment in cisplatin-ineligible patients with locally advanced and metastatic urothelial carcinoma: a single-arm, multicentre, phase 2 trial. Lancet. 2017;389(10064):67-76.

3. Borghaei $\mathrm{H}$, et al. Nivolumab versus Docetaxel in advanced nonsquamous non-small-cell lung cancer. N Engl J Med. 2015;373(17):1627-39.

4. Brahmer J, et al. Nivolumab versus Docetaxel in advanced squamous-cell non-small-cell lung cancer. N Engl J Med. 2015;373(2):123-35.

5. Fehrenbacher $L$, et al. Atezolizumab versus docetaxel for patients with previously treated non-small-cell lung cancer (POPLAR): a multicentre, openlabel, phase 2 randomised controlled trial. Lancet. 2016:387(10030):1837-46.

6. Ferris RL, et al. Nivolumab for recurrent squamous-cell carcinoma of the head and neck. N Engl J Med. 2016;375(19):1856-67.

7. Herbst RS, et al. Pembrolizumab versus docetaxel for previously treated, PDL1-positive, advanced non-small-cell lung cancer (KEYNOTE-010): a randomised controlled trial. Lancet. 2016;387(10027):1540-50.

8. Kaufman $\mathrm{HL}$, et al. Avelumab in patients with chemotherapy-refractory metastatic Merkel cell carcinoma: a multicentre, single-group, open-label, phase 2 trial. Lancet Oncol. 2016;17(10):1374-85.

9. Langer $\mathrm{CJ}$, et al. Carboplatin and pemetrexed with or without pembrolizumab for advanced, non-squamous non-small-cell lung cancer: a randomised, phase 2 cohort of the open-label KEYNOTE-021 study. Lancet Oncol. 2016;17(11):1497-508.

10. Motzer RJ, et al. Nivolumab versus Everolimus in advanced renal-cell carcinoma. N Engl J Med. 2015;373(19):1803-13.

11. Reck M, et al. Pembrolizumab versus chemotherapy for PD-L1-positive nonsmall-cell lung cancer. N Engl J Med. 2016;375(19):1823-33.

12. Ribas A, et al. Pembrolizumab versus investigator-choice chemotherapy for ipilimumab-refractory melanoma (KEYNOTE-002): a randomised, controlled, phase 2 trial. Lancet Oncol. 2015;16(8):908-18.

13. Rittmeyer $\mathrm{A}$, et al. Atezolizumab versus docetaxel in patients with previously treated non-small-cell lung cancer (OAK): a phase 3, open-label, multicentre randomised controlled trial. Lancet. 2017;389(10066):255-65.

14. Robert C, et al. Nivolumab in previously untreated melanoma without BRAF mutation. N Engl J Med. 2015;372(4):320-30.

15. Weber JS, et al. Nivolumab versus chemotherapy in patients with advanced melanoma who progressed after anti-CTLA-4 treatment (CheckMate 037): a randomised, controlled, open-label, phase 3 trial. Lancet Oncol. 2015;16(4):375-84

16. Younes A, et al. Nivolumab for classical Hodgkin's lymphoma after failure of both autologous stem-cell transplantation and brentuximab vedotin: a multicentre, multicohort, single-arm phase 2 trial. Lancet Oncol. 2016;17(9):1283-94.

17. FDA Approved Drug Products [Internet]. Available from: http://www. accessdata.fda.gov/scripts/cder/daf. [cited 2017 May 1].

18. Choueiri TK, Motzer RJ. Systemic therapy for metastatic renal-cell carcinoma. N Engl J Med. 2017;376(4):354-66.

19. Ott PA, Hodi FS, Robert C. CTLA-4 and PD-1/PD-L1 blockade: new immunotherapeutic modalities with durable clinical benefit in melanoma patients. Clin Cancer Res. 2013;19(19):5300-9.

20. Kim JM, Chen DS. Immune escape to PD-L1/PD-1 blockade: seven steps to success (or failure). Ann Oncol. 2016;27(8):1492-504.

21. Sharma $P$, et al. Primary, adaptive, and acquired resistance to cancer immunotherapy. Cell. 2017;168(4):707-23. 
22. Zaretsky JM, et al. Mutations associated with acquired resistance to PDblockade in melanoma. N Engl J Med. 2016;375(9):819-29.

23. Hugo W, et al. Genomic and Transcriptomic features of response to anti-PD1 therapy in metastatic melanoma. Cell. 2016;165(1):35-44.

24. Lipson EJ, et al. Durable cancer regression off-treatment and effective reinduction therapy with an anti-PD-1 antibody. Clin Cancer Res. 2013;19(2):462-8.

25. Lee JY, et al. Structural basis of checkpoint blockade by monoclonal antibodies in cancer immunotherapy. Nat Commun. 2016;7:13354.

26. Curran MA, et al. PD-1 and CTLA-4 combination blockade expands infiltrating $T$ cells and reduces regulatory $T$ and myeloid cells within B16 melanoma tumors. Proc Natl Acad Sci U S A. 2010;107(9):4275-80.

27. Bellone $M$, Calcinotto $A$. Ways to enhance lymphocyte trafficking into tumors and fitness of tumor infiltrating lymphocytes. Front Oncol. 2013;3:231.

28. Shindo $Y$, et al. Combination immunotherapy with 4-1BB activation and PD1 blockade enhances antitumor efficacy in a mouse model of subcutaneous tumor. Anticancer Res. 2015;35(1):129-36.

29. Lu L, et al. Combined PD-1 blockade and GITR triggering induce a potent antitumor immunity in murine cancer models and synergizes with chemotherapeutic drugs. J Transl Med. 2014;12:36.

30. Guo Z, et al. PD-1 blockade and OX40 triggering synergistically protects against tumor growth in a murine model of ovarian cancer. PLoS One. 2014;9(2):e89350.

31. West EE, et al. PD-L1 blockade synergizes with IL-2 therapy in reinvigorating exhausted T cells. J Clin Invest. 2013;123(6):2604-15.

32. Sharabi $A B$, et al. Stereotactic radiation therapy augments antigen-specific PD-1-mediated antitumor immune responses via cross-presentation of tumor antigen. Cancer Immunol Res. 2015;3(4):345-55.

33. Kaufman $\mathrm{HL}$, et al. The use of registries to improve cancer treatment: a National Database for patients treated with interleukin-2 (IL-2). J Pers Med. 2014;4(1):52-64.

\section{Submit your next manuscript to BioMed Central} and we will help you at every step:

- We accept pre-submission inquiries

- Our selector tool helps you to find the most relevant journal

- We provide round the clock customer support

- Convenient online submission

- Thorough peer review

- Inclusion in PubMed and all major indexing services

- Maximum visibility for your research

Submit your manuscript at www.biomedcentral.com/submit 